\title{
Riesgo diferencial de hemorragia intracraneana en pacientes con fibrilación auricular no valvular con el uso de nuevos anticoagulantes orales vs. warfarina. Revisión sistemática de la literatura y análisis de subpoblaciones
}

Differential Risk of Intracranial Hemorrhage in Patients with Nonvalvular Atrial Fibrillation with Usage of Novel Oral Anticoagulants vs. Warfarin. Systematic Review and Analysis of Subpopulations

Samir Alejandro Aruachán Torres Pontificia Universidad Javeriana, Colombia

Ana María Ayala Copete

Pontificia Universidad Javeriana, Colombia

Daniela Patino-Hernandez

Pontificia Universidad Javeriana, Colombia

Julián David Ríos-Zuluaga

Pontificia Universidad Javeriana, Colombia Ángel Alberto García Peña

Pontificia Universidad Javeriana, Colombia

Carlos Alberto Cano-Gutiérrez

Pontificia Universidad Javeriana, Colombia

Cómo citar: Aruachán Torres SA, Ayala Copete AM, Patino-Hernandez D, Ríos-Zuluaga JD, García Peña ÁA, Cano-Gutiérrez CA. Riesgo diferencial de hemorragia intracraneana en pacientes con fibrilación auricular no valvular con el uso de nuevos anticoagulantes orales vs. Warfarina: revisión sistemática de la literatura y análisis de subpoblaciones. Univ Med. 2017;58(2):1-9. doi: https://doi.org/10.11144/Javeriana.umed58-2.warf

\section{RESUMEN}

Introducción: La anticoagulación en pacientes con fibrilación auricular no valvular previene eventos tromboembólicos con incremento implícito en el riesgo de complicaciones hemorrágicas, principalmente la hemorragia intracraneana. Métodos: Se realizó una revisión sistemática de la literatura del periodo 2011-2016 en bases de datos (PubmedEmbase). Se incluyeron metanálisis, revisiones sistemáticas y ensayos clínicos aleatorizados de las moléculas de interés (apixabán, dabigatrán, edoxabán, rivaroxabán). Se identificaron 512 artículos, tras lo cual se seleccionaron catorce estudios para el análisis final. Resultados: Durante la evaluación comparativa de las cuatro moléculas en diferentes dosis versus warfarina se evidenció una menor prevalencia de hemorragia intracraneana en pacientes manejados con nuevos anticoagulantes orales; en todos los artículos evaluados, los resultados apoyan su uso. Sin embargo, al evaluar desenlaces hemorrágicos en el caso de subpoblaciones, los resultados parecen tener menor impacto y, en algunos casos, no logran la significancia estadística y se encontró reducción o desaparición del beneficio. Conclusiones: Los resultados pueden aportar herramientas de utilidad que apoyarían al clínico en la toma de decisiones acerca de la elección del tipo de anticoagulante en escenarios clínicos específicos. 
Palabras clave

fibrilación auricular; warfarina; anticoagulantes; hemorragia cerebral.

\begin{abstract}
Introduction: Patients with non-valvular atrial fibrillation require anticoagulation therapy for prevention of thromboembolic events. The safest type of anticoagulation therapy regarding intracranial hemorrhage has not been established yet. Methods: We conducted a systematic review of literature in the time period 2011-2016 in databases (PubMed-EMBASE). We included metaanalysis, systematic reviews and randomized clinical trials addressing the molecules of interest (apixaban, dabigatran, edoxaban, rivaroxaban). We identified 512 articles, after which 14 studies were selected for the final analysis. Results: Through comparative evaluation of the four molecules in different doses versus warfarin, we found a decrease in intracranial hemorrhage in patients on therapy with novel anticoagulants, results support their usage in all of the articles included for analysis. However, when evaluating hemorrhage-related outcomes in different subpopulations, the results seem to have a smaller impact and in some cases they do not reach statistical significance, also, the benefit was reduced or disappeared. Conclusions: The results from this review can serve as useful tools in order to support physicians in the decision-making process regarding choice of the right type of anticoagulant therapy in specific clinical situations.

Keywords

atrial fibrillation; warfarin; anticoagulants; intracranial hemorrhages.
\end{abstract}

\section{Introducción}

La fibrilación auricular (FA) es una taquiarritmia supraventricular, caracterizada por una descarga y activación desorganizada y rápida de las aurículas de entre 400 y 700 ciclos por minuto, cuya consecuencia es una alteración en la función biomecánica auricular [1]. Su patrón típico de aparición en el electrocardiograma es el de la ausencia de ondas p, que preceden cada complejo QRS, y en lugar de esto, la presencia de las denominadas ondas fibrilatorias (ondas f), variables en forma, tamaño e intervalo [1].

Hoy en día, la FA es la arritmia sostenida más frecuente en el mundo [2]. Aumenta su incidencia con la edad y su prevalencia está alrededor del $10 \%$ en personas mayores de 80 años de edad. Se estima que casi un cuarto de la población mundial tendrá FA en su vida [1]. Tradicionalmente, para su manejo se han utilizado con éxito medicamentos anticoagulantes para prevenir eventos tromboembólicos; sin embargo, lo anterior representa un gran reto clínico, debido a que estos confieren, al mismo tiempo, un incremento en el riesgo de hemorragia y la complicación más temida es el sangrado del sistema nervioso central, tomando en cuenta que es un generador importante de discapacidad y se asocia con incremento en costos de atención y mortalidad.

Se ha demostrado que los pacientes que se encuentran en terapia con anticoagulantes orales (ACO) de intensidades convencionales pueden incrementar el riesgo de hemorragia intracraneana de 7 a 10 veces, a una tasa absoluta de alrededor del $1 \%$ por año de uso [3]. En la última década, y debido a la necesidad de nuevas terapias con distintas dianas terapéuticas (inhibiendo directa o indirectamente el factor Xa o la trombina) con un perfil farmacológico más seguro y eficaz en comparación con la warfarina como tratamiento de referencia, particularmente en lo referente al monitoreo, dosificación, interacciones farmacológicas y tiempo en rango terapéutico, surge un nuevo grupo de anticoagulantes no vitamino-K-dependientes. Estas nuevas terapias anticoagulantes están aprobadas para pacientes con fibrilación auricular no valvular (FANV), término acuñado para describir la FA no relacionada con valvulopatías severas, particularmente de la válvula mitral o presencia de prótesis valvular. Esta definición, que varía entre los diferentes estudios pivotales, se explica en la tabla 1 . Existen cuatro medicamentos aprobados por las agencias regulatorias internacionales para su uso en pacientes con FANV. En la tabla 2 se muestran los aspectos más relevantes de su farmacología y estudios que aprueban su uso en esta condición. 
Tabla 1. Tipos de fibrilación auricular

\begin{tabular}{|l|l|}
\hline \multicolumn{1}{|c|}{ Término } & \multicolumn{1}{c|}{ Definición } \\
\hline Fibrilación auricular paroxística & $\begin{array}{l}\text { Se resuelve espontáneamente o es tratada en } \\
7 \text { días. Los episodios pueden recurrir con } \\
\text { una frecuencia variable }\end{array}$ \\
\hline Fibrilación auricular persistente & $\begin{array}{l}\text { Fibrilación auricular continua con duración } \\
\text { mayor de 7 días. }\end{array}$ \\
\hline $\begin{array}{l}\text { Fibrilación auricular persistente de larga } \\
\text { data }\end{array}$ & $\begin{array}{l}\text { Fibrilación auricular continua de más de 12 } \\
\text { meses. }\end{array}$ \\
\hline Fibrilación auricular permanente & $\begin{array}{l}\text { Término utilizado cuando se requieren } \\
\text { intervenciones adicionales para recuperar o } \\
\text { mantener el ritmo sinusal. }\end{array}$ \\
\hline Fibrilación auricular no valvular & $\begin{array}{l}\text { Fibrilación auricular en ausencia de } \\
\text { estenosis mitral reumática, válvula mecánica } \\
\text { o bioprotésica o valvuloplastia mitral. }\end{array}$ \\
\hline
\end{tabular}

Fuente: adaptado de January CT, Wann LS, Alpert JS, Calkins H, Cigarroa JE, Cleveland J Joseph C, et al. AHA/ACC/HRS Guideline for the management of patients with atrial fibrillation: executive summary: A Report of the American College of Cardiology/American Heart Association Task Force on Practice Guidelines and the Heart Rhythm Society. J Am Coll Cardiol. 2014 Dec 2;64(21):2246-80.

Tabla 2. Fármacos aprobados en el manejo de FANV

\begin{tabular}{|c|c|c|c|c|}
\hline Fármaco/característica & Dabigatrán & Rivaroxabán & Apixabán & Edoxabán \\
\hline Diana farmacológica & Trombina & Factor Xa & Factor Xa & Factor Xa \\
\hline Absorción & $\begin{array}{l}\text { Todo el tracto } \\
\text { gastrointestinal }\end{array}$ & \begin{tabular}{|l|} 
Estómago \\
principalmente
\end{tabular} & $55 \%$ intestinal & $\begin{array}{l}\text { No } \\
\text { información }\end{array}$ \\
\hline Biodisponibilidad (\%) & 6 & 80 & 50 & 50 \\
\hline Vida media (h) & 12 a 17 & 7 a 11 & 9 a 14 & 9 a 11 \\
\hline Unión a proteínas (\%) & 35 & 95 & 87 & 54 \\
\hline Excreción renal (\%) & 80 & 66 & 25 & 35 \\
\hline Posología & dos/día & uno/día & dos/día & uno/día \\
\hline Presentación & cápsula & tableta & tableta & tableta \\
\hline $\begin{array}{l}\text { Estudio que valida su uso } \\
\text { (tipo de estudio) }\end{array}$ & $\begin{array}{l}\text { RE-LY } \\
\text { (ensayo clínico } \\
\text { aleatorizado } \\
\text { doble ciego } \\
\text { multicéntrico) }\end{array}$ & $\begin{array}{l}\text { ROCKET AF } \\
\text { (ensayo clínico } \\
\text { aleatorizado } \\
\text { doble ciego } \\
\text { multicéntrico) }\end{array}$ & $\begin{array}{l}\text { ARISTOTLE } \\
\text { (ensayo clínico } \\
\text { aleatorizado } \\
\text { doble ciego } \\
\text { multicéntrico) }\end{array}$ & \begin{tabular}{|l|} 
ENGAGE-AF- \\
TIMI (ensayo \\
clínico \\
aleatorizado \\
doble ciego \\
multicéntrico) \\
\end{tabular} \\
\hline
\end{tabular}

Fuente: adaptado de Veiga Fernández F, Malfeito Jiménez M del R, Barros Cerviño SM, Magariños Losada M del M. La anticoagulación oral en el anciano con fibrilación auricular no valvular. Rev Esp Geriatr Gerontol. 2015;50(3):134-42.

Tomando en cuenta lo anterior, se muestran los hallazgos tras la realización de un análisis sistemático de la literatura para evaluar la incidencia de hemorragia intracraneana en pacientes tratados con ACO dependientes de vitamina $\mathrm{K}$ (warfarina) y los nuevos anticoagulantes orales (NACO): rivaroxabán, apixabán, edoxabán y dabigatrán .

\section{Materiales y métodos}

Tipo de estudio y población

Se realizó una revisión sistemática de la literatura científica en pacientes con FANV y terapia anticoagulante que evaluara el desarrollo de hemorragia intracraneana.

\section{Definición del desenlace}

El desenlace seleccionado en los estudios fue hemorragia intracraneana, entendida como accidente cerebrovascular hemorrágico, hemorragia intraventricular, subaracnoidea, subdural, epidural e intraparenquimatosa, diagnosticada con base en los hallazgos clínicos e imagenológicos respectivos.

Criterios de inclusión, exclusión y restricciones empleadas en la búsqueda

Búsqueda de la literatura. Se realizó una búsqueda de la literatura en Pubmed incluyendo los siguientes términos: atrial fibrillation, rivaroxaban, dabigatran, edoxabán, apixaban, warfarin, intracranial haemorrhage, intracranial hemorrhages, major bleeding, hemorrhage, haemorrhage, bleeding, subarachnoid hemorrhage, cerebral hemorrhage, intracerebral hemorrhage, intraventricular hemorrhage, subdural hemorrhage y stroke.

([atrial fibrillation $\{\mathrm{MeSH}\}]$ AND [rivaroxaban] OR [rivaroxaban $\{\mathrm{MeSH}\}$ ] $\mathrm{OR}$ [dabigatran] OR [dabigatran $\{\mathrm{MeSH}\}]$ OR [edoxaban] OR [apixaban]) AND (warfarin $\{\mathrm{MeSH}\})$ AND ([intracranial hemorrhage] OR [intracranial hemorrhages $\{\mathrm{MeSH}\}$ ] OR [major bleeding] OR [hemorrhage $\{\mathrm{MeSH}\}]$ OR [hemorrhage] OR [bleeding] OR [subarachnoid hemorrhage $\{\mathrm{MeSH}\}]$ OR [cerebral hemorrhage $\{\mathrm{MeSH}\}]$ OR [intracerebral hemorrhage] OR [intraventricular hemorrhage] OR [subdural hemorrhage] OR [stroke $\{\mathrm{MeSH}\}])$.

Criterios de inclusión:

Ensayos clínicos aleatorizados. 
Revisiones sistemáticas.

Metanálisis.

Artículos que evaluaran uno o más de los desenlaces descritos, de manera comparativa con warfarina vs. NACO.

Criterios de exclusión:

Uso concomitante de terapia antiagregante o anticoagulante no oral.

Pacientes menores de 18 años de edad.

Restricciones empleadas en la búsqueda: se limitó la búsqueda de la literatura a humanos, metanálisis, revisión sistemática y ensayo clínico aleatorizado, publicaciones en los últimos tres años y restricción de idiomas a español e inglés.

\section{Selección y evaluación de artículos}

De acuerdo con los criterios de búsqueda establecidos y con los términos MeSH empleados, la búsqueda arrojó un total de 512 artículos en PubMed. Se excluyeron 424 artículos tras la aplicación de límites. Posteriormente, se eliminaron 68 artículos por no considerarlos pertinentes o apropiados para la resolución de la pregunta de investigación para un total de 20 artículos que se sometieron a revisión metodológica de acuerdo a los criterios de la Scottish Intercollegiate Guidelines Network (SIGN).

Para la evaluación de los artículos preseleccionados se utilizaron las listas de comprobación SIGN para revisiones sistemáticas y metaanálisis y para ensayos clínicos aleatorizados, con el objetivo de cumplir los estándares conceptuales y metodológicos para la adecuada presentación de la revisión sistemática.

Una vez llevado a cabo el proceso de evaluación de calidad y de la preselección, así como de excluir aquellos análisis secundarios de estudios, se obtuvo un número final de 14 artículos empleados en el presente trabajo.

Para analizar los artículos seleccionados, se clasificaron en dos grupos principales: aquellos que evaluaran los desenlaces de interés en población general y aquellos que los evaluaran en subgrupos poblacionales. Posteriormente, se discriminaron los artículos según desenlace evaluado.

\section{Resultados}

\section{Población general}

\section{Hemorragia intracraneana}

Se encontraron 6 artículos que evaluaron hemorragia intracraneana con el uso de nuevos anticoagulantes orales versus warfarina. El estudio de Granger et al. [4] realizó una evaluación comparativa entre apixabán y warfarina que evidenció menor riesgo con el uso de apixabán (HR: 0,42; IC $95 \%$ : 0,30-0,58; $\mathrm{p}<0,001)$. El artículo de Connoly et al. [5] reportó menor riesgo con el uso de dabigatrán de $110 \mathrm{mg}$ vs. warfarina (HR: 0,31; IC 95 \%: 0,20-0,47; p < 0,001) y dabigatrán de $150 \mathrm{mg}$ (HR: 0,40; IC $95 \%$ : 0,27-0,60; p < $0,001)$. Patel et al. [6] llevaron a cabo una evaluación comparativa entre rivaroxabán y warfarina, que reveló menor riesgo con el uso de rivaroxabán (HR: 0,67: IC 95\%: 0,47-0,93; $\mathrm{p}<0,02)$. Giugliano et al. [7] reportaron menor riesgo de hemorragia intracraneana con el uso de edoxabán de $60 \mathrm{mg}$ (HR: 0,47; IC $95 \%$ : 0,34-0,63; p < 0,001) y edoxabán de $30 \mathrm{mg}$ (HR: 0,30; IC $95 \%$ : 0,21-0,43; p < $0,001)$ al compararlo con warfarina. Capodanno et al. [8] constrastaron apixabán, rivaroxabán o dabigatrán versus warfarina, y hallaron menor riesgo con comparación con el uso de warfarina (HR: 0,46; IC $95 \%$ : 0,33-0,65; p $<0,001$ ). Finalmente, el estudio de Mitchell et al. [9] reportó menor riesgo con el uso de apixabán (HR: 0,41; IC $95 \%$ : 0,29-0,57), y no diferencia estadísticamente con rivaroxabán (HR: 0,71; IC $95 \%: 0,51-1,01)$ y reducción significativa con el uso de dabigatrán de $150 \mathrm{mg}$ (HR: 0,42; IC $95 \%$ : 0,28-0,61) o dabigatrán de $110 \mathrm{mg}$ (HR: 0,30; IC $95 \%$ : 0,19-0,46). Estos datos se pueden encontrar en la tabla 3. 
Tabla 3. Hemorragia intracraneana en población general

\begin{tabular}{|c|c|c|c|c|c|c|c|}
\hline \multirow[t]{2}{*}{ Artículo } & \multirow[t]{2}{*}{ Comparador } & \multicolumn{4}{|c|}{ Medidas estadísticas } & \multicolumn{2}{|c|}{ Eventos del total (\%) } \\
\hline & & HR & IC 9 & $5 \%$ & $p$ & NACO & Warfarina \\
\hline $\begin{array}{l}\text { Granger et al., } \\
2011|5|\end{array}$ & Apixabán & 0,42 & 0,30 & 0,58 & $<0,001$ & 0,33 & 0,80 \\
\hline \multirow[t]{2}{*}{$\begin{array}{l}\text { Connolly et al., } \\
2009[6]\end{array}$} & $\begin{array}{l}\text { Dabigatrán de } 110 \\
\text { mg }\end{array}$ & 0,31 & 0,20 & 0,47 & $<0,001$ & 0,23 & \multirow[t]{2}{*}{0,74} \\
\hline & $\begin{array}{l}\text { Dabigatrán de } 150 \\
\text { mg }\end{array}$ & 0,40 & 0,27 & 0,60 & $<0,001$ & 0,30 & \\
\hline $\begin{array}{l}\text { Patel et al., } 2011 \\
\text { [7] }\end{array}$ & Rivaroxabán & 0,67 & 0,47 & 0,93 & 0,02 & 0,8 & 2,2 \\
\hline \multirow{2}{*}{$\begin{array}{l}\text { Giugliano ct al., } \\
2013|8|\end{array}$} & Edoxabán de $60 \mathrm{mg}$ & 0,47 & 0,34 & 0,63 & $<0,001$ & 0,39 & \multirow[t]{2}{*}{0,85} \\
\hline & Edoxabán de $30 \mathrm{mg}$ & 0,30 & 0,21 & 0,43 & $<0,001$ & 0,26 & \\
\hline $\begin{array}{l}\text { Capodanno et al., } \\
2012[9]\end{array}$ & $\begin{array}{l}\text { Apixabán, } \\
\text { rivaroxabán y } \\
\text { dabigatrán }\end{array}$ & 0,46 & 0,33 & 0,65 & $<0,001$ & 0,6 & 1,3 \\
\hline \multirow{4}{*}{$\begin{array}{l}\text { Mitchell et al., } \\
2013[10]\end{array}$} & Apixabán & 0,41 & 0,29 & 0,57 & & & \\
\hline & Rivaroxabán & 0,71 & 0,51 & 1,01 & & & \\
\hline & $\begin{array}{l}\text { Dabigatrán de } 150 \\
\text { mg }\end{array}$ & 0,42 & 0,28 & 0,61 & & & \\
\hline & $\begin{array}{l}\text { Dabigatrán de } 110 \\
\mathrm{mg}\end{array}$ & 0,30 & 0,19 & 0,46 & & & \\
\hline
\end{tabular}

NACO: nuevos anticoagulantes orales.

\section{Accidente cerebrovascular hemorrágico}

Se encontraron cinco artículos que evaluaron la hemorragia intracraneana con el uso de nuevos anticoagulantes orales versus warfarina. El artículo de Granger et al. [4] mostró disminución del riesgo de accidente cerebrovascular hemorrágico $(\mathrm{ACVH})$ con el uso de apixabán (HR: 0,51; IC 95\%: 0,35-0,75; $\mathrm{p}<0,001)$. Connolly et al. [5] evidenciaron menor riesgo de $\mathrm{ACVH}$ con el uso de dabigatrán de $110 \mathrm{mg}$ (HR: 0,31; IC 95\%: 0,17-0,56; p $<0,001)$ y dabigatrán de $150 \mathrm{mg}$ (HR: 0,26; IC $95 \%$ : 0,14-0,49; $<<0,001)$. Giugliano et al. [7] reportaron menor riesgo hemorrágico con el uso edoxabán de $60 \mathrm{mg}$ (HR: 0,54; IC $95 \%$ : 0,38-0,77; p < 0,001) y edoxabán de 30 mg (HR: 0,33; IC $95 \%$ : 0,22-0,50; p < 0,001). En el estudio de Capodanno et al. [8] se evidenció menor riesgo de ACVH con el uso de apixabán, rivaroxabán o dabigatrán (HR: 0.45; IC $95 \%$ : 0,35-0,58; $<<0,001)$. Mitchell et al. [9] realizaron una evaluación comparativa entre apixabán, rivaroxabán o dabigatrán versus warfarina y observaron menor con apixabán (HR: 0.51; IC $95 \%$ : 0,35-0,75), rivaroxabán (HR: 0,58; IC $95 \%$ : 0,38-0,89), dabigatrán de $150 \mathrm{mg}$ (HR: 0,26; IC $95 \%$ : 0,13-0,48) y dabigatrán de $110 \mathrm{mg}$ (HR: 0,31; IC $95 \%$ : 0,16-0,56). Esta información se encuentra en la tabla 4.
Tabla 4. Accidente cerebrovascular hemorrágico en población general

\begin{tabular}{|c|c|c|c|c|c|c|c|}
\hline \multirow{2}{*}{ Artículo } & \multirow[t]{2}{*}{ Comparador } & \multicolumn{4}{|c|}{ Medidas estadísticas } & \multicolumn{2}{|c|}{ Eventos del total (\%) } \\
\hline & & HR & IC 9 & $5 \%$ & $p$ & NACO & Warfarina \\
\hline $\begin{array}{l}\text { Granger et } \\
\text { al., 2011 [5] }\end{array}$ & Apixabán & 0,51 & 0,35 & 0,75 & $<0,001$ & 0,24 & 0,47 \\
\hline \multirow{2}{*}{$\begin{array}{l}\text { Connolly et } \\
\text { al., } 2009 \text { [6] }\end{array}$} & Dabigatrán de $110 \mathrm{mg}$ & 0,31 & 0,17 & 0,56 & $<0,001$ & 0,38 & \multirow[t]{2}{*}{0,12} \\
\hline & Dabigatrán de $150 \mathrm{mg}$ & 0,26 & 0,14 & 0,49 & $<0,001$ & 0,10 & \\
\hline \multirow{2}{*}{$\begin{array}{l}\text { Giugliano et } \\
\text { al., } 2013[8]\end{array}$} & Edoxabán de $60 \mathrm{mg}$ & 0,54 & 0,38 & 0,77 & $<0,001$ & 0,26 & \multirow[t]{2}{*}{0,47} \\
\hline & Edoxabán de $30 \mathrm{mg}$ & 0,33 & 0,22 & 0,50 & $<0,001$ & 0,16 & \\
\hline $\begin{array}{l}\text { Capodanno } \\
\text { et al., } 2012 \\
{[9]}\end{array}$ & $\begin{array}{l}\text { Apixabán, rivaroxabán y } \\
\text { dabigatrán }\end{array}$ & 0,45 & 0,35 & 0,58 & $<0,001$ & 0,3 & 0,8 \\
\hline \multirow{4}{*}{$\begin{array}{l}\text { Mitchell et } \\
\text { al., } 2013 \text { [10] }\end{array}$} & Apixabán & 0,51 & 0,35 & 0,75 & & & \\
\hline & Rivaroxab: & 0,58 & 0,38 & 0,89 & & & \\
\hline & Dabigatrán de $150 \mathrm{mg}$ & 0,26 & 0,13 & 0,48 & & & \\
\hline & Dabigatrán de $110 \mathrm{mg}$ & 0,31 & 0,16 & 0,56 & & & \\
\hline
\end{tabular}

NACO:nuevos anticoagulantes orales.

\section{Hemorragia intracraneana fatal}

Se encontró un estudio que evalúa este desenlace, que es el de Giugliano et al. [7], el cual reportó menor riesgo de hemorragia intracraneana fatal con el uso de edoxabán de 60 mg en comparación con warfarina (HR: 0,58; IC $95 \%: 0,35-0,95 ; \mathrm{p}=0,03)$ y edoxabán de $30 \mathrm{mg}$ (HR: 0,28; IC 95 \%: 0,15-0,53; p < 0,001). Estos datos se encuentran en la tabla 5 .

Tabla 5. Hemorragia intracraneana fatal en población general

\begin{tabular}{|c|c|c|c|c|c|c|c|}
\hline \multirow{2}{*}{ Articulo } & \multirow{2}{*}{ Comparador } & \multicolumn{4}{|c|}{ Medidas estadisticas } & \multicolumn{2}{|c|}{ Eventos del total $(\%)$} \\
\hline & & $\mathrm{HR}$ & & & $p$ & & \\
\hline $\begin{array}{l}\text { Civiuglano } \\
2013 \text { (81 }\end{array}$ & 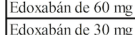 & $\begin{array}{l}0.58 \\
0.28 \\
\end{array}$ & $\frac{0,3}{0.1}$ & 0,95 & $\frac{0,02}{<0,000}$ & $\frac{0,15}{0,08}$ & \\
\hline
\end{tabular}

NACO:nuevos anticoagulantes orales.

\section{Subgrupos poblacionales: población asiática}

\section{Hemorragia intracraneana}

Dos estudios evaluaron el desenlace de hemorragia intracraneana en población asiática. El artículo de Hori et al. [10] reportó menor riesgo de hemorragia con el uso de dabigatrán de $110 \mathrm{mg}$ (HR: 0,20; IC $95 \%$ : 0,07-0,60; *p: 0,46) y dabigatrán de $150 \mathrm{mg}$ (HR: 0,40; IC $95 \%$ : 0,18-0,92; *p: 0,95) en comparación con warfarina. El estudio de Goto et al. [11] reportó menor riesgo de hemorragia intracraneana con apixabán (HR: 0,36; IC 95 \%: 0,18-0,71; *p: $0,60)$. Ambos estudios derivados de subanálisis del estudio pivotal para cada una de las moléculas descritas (*p para interacción). La información descrita se encuentra en la tabla 6. 
Tabla 6. Hemorragia intracraneana en población asiática

\begin{tabular}{|c|c|c|c|c|c|c|c|}
\hline \multirow[t]{2}{*}{ Artículo } & \multirow[t]{2}{*}{ Comparador } & \multicolumn{4}{|c|}{ Medidas estadísticas } & \multicolumn{2}{|c|}{$\begin{array}{c}\text { Eventos del total } \\
(\%)\end{array}$} \\
\hline & & HR & \multicolumn{2}{|c|}{ IC $95 \%$} & $\begin{array}{c}\text { p para } \\
\text { interacción }\end{array}$ & NACO & Warfarina \\
\hline \multirow{4}{*}{$\begin{array}{l}\text { Hori et } \\
\text { al., 2013 } \\
{[11]}\end{array}$} & $\begin{array}{l}\text { Dabigatran de } 110 \\
\text { mg en asiáticos }\end{array}$ & 0,20 & 0,07 & 0,60 & 0,46 & 0,23 & 1,10 \\
\hline & $\begin{array}{l}\text { Dabigatrán de } 110 \\
\text { mg en no } \\
\text { asiáticos }\end{array}$ & 0,32 & 0,20 & 0,51 & 0,46 & 0,23 & 0,71 \\
\hline & $\begin{array}{l}\text { Dabigatrán de } 150 \\
\text { mg en asiáticos }\end{array}$ & 0,40 & 0,18 & 0,92 & 0,95 & 0,45 & 1,10 \\
\hline & $\begin{array}{l}\text { Dabigatrán de } 150 \\
\text { mg en no } \\
\text { asiáticos }\end{array}$ & 0,41 & 0,27 & 0,63 & 0,95 & 0,29 & 0,71 \\
\hline \multirow{2}{*}{$\begin{array}{l}\text { Goto et } \\
\text { al., 2014 } \\
{[12]}\end{array}$} & $\begin{array}{l}\text { Apixabán en } \\
\text { asiáticos del este }\end{array}$ & 0,359 & 0,180 & 0,714 & 0,5953 & 0,67 & 1,88 \\
\hline & $\begin{array}{l}\text { Apixabán en no } \\
\text { asiáticos del este }\end{array}$ & 0,440 & 0,304 & 0,636 & 0,5953 & 0,30 & 0,67 \\
\hline
\end{tabular}

NACO:nuevos anticoagulantes orales.

\section{Accidente cerebrovascular hemorrágico}

Se encontraron dos artículos que evaluaron accidente cerebrovascular hemorrágico en población asiática. El estudio de Hori et al. [10] reportó mayor riesgo de ACVH con el uso de warfarina que con dabigatrán de 110 mg (HR: 0,15; IC $95 \%: 0,03-0,66$; *p: 0,27) y dabigatrán de $150 \mathrm{mg}$ (HR: 0,22; IC $95 \%$ : 0,06-0,77; p: 0,76). Ambos estudios se derivaron del subanálisis del estudio pivotal para cada una de las moléculas descritas ("p para interacción). Estos datos se encuentran en la tabla 7.

Tabla 7. Accidente cerebrovascular hemorrágico en población asiática

\begin{tabular}{|c|c|c|c|c|c|c|c|}
\hline \multirow[b]{2}{*}{ Articulo } & \multirow[b]{2}{*}{ Comparador } & \multicolumn{4}{|c|}{ Medidas estadísticas } & \multicolumn{2}{|c|}{ Eventos del total $(\%)$} \\
\hline & & \multirow{2}{*}{\begin{tabular}{|l|} 
HR \\
0,15 \\
\end{tabular}} & \multicolumn{2}{|c|}{ IC $95 \%$} & \multirow{2}{*}{\begin{tabular}{|c}
$\begin{array}{c}\text { p para } \\
\text { interacción }\end{array}$ \\
0,27
\end{tabular}} & \multirow{2}{*}{$\begin{array}{c}\text { NACO } \\
0,11 \\
\end{array}$} & \multirow{2}{*}{\begin{tabular}{|c|} 
Warfarinin: \\
0,75 \\
\end{tabular}} \\
\hline \multirow{4}{*}{$\begin{array}{l}\text { Hori et al., } \\
2013[11]\end{array}$} & $10 \mathrm{mg}$ en asiáticos & & 0,03 & 0,66 & & & \\
\hline & $\begin{array}{l}\text { Dabigatán de } 110 \text { mg en no } \\
\text { asiáticos }\end{array}$ & 0,37 & 0,19 & 0,72 & 0,27 & 0,12 & 0,32 \\
\hline & Dabigatrán de 15 & 0,22 & 0,06 & 0,77 & 0,76 & 0,17 & 0,75 \\
\hline & $\begin{array}{l}\text { Dabigataŕn de } 150 \mathrm{mg} \text { en no } \\
\text { asiäticos }\end{array}$ & 0,28 & 0,13 & 0,58 & 0,76 & 0,09 & 0,32 \\
\hline et al., & Apixabán en asiáticos del este & 0,253 & 0,103 & 0,618 & 0, & 17 & 1,33 \\
\hline 411 & Apixabán en no asiáticos del este & 0,623 & 0,406 & 0,957 & 0,0745 & 0,23 & 0,36 \\
\hline
\end{tabular}

NACO:nuevos anticoagulantes orales.

\section{Subgrupos poblacionales: adulto mayor}

\section{Hemorragia intracraneana}

Se encontraron dos artículos que evaluaron hemorragia intracraneana con el uso de nuevos anticoagulantes orales versus warfarina en el adulto mayor. El estudio de Halperin et al. [12] realizó una evaluación comparativa entre rivaroxabán y warfarina y evidenció menor riesgo con el uso de rivaroxabán (HR: 0,80; IC 95 \%: 0,50-1,28; *p: 0,27). El artículo de Halvorsen et al. [13] reportó menor riesgo con el uso de apixabán en pacientes con edad entre 65 y 75 años (HR: 0,35; IC $95 \%$ : 0,20-0,60; *p: 0,2) y apixabán en mayores de 75 años de edad (HR: 0,34; IC 95 \%: 0,20-0,57; *p: 0,2). *p para interacción. Esta información se encuentra en la tabla 8.

Tabla 8. Hemorragia intracraneana en el adulto mayor

\begin{tabular}{|c|c|c|c|c|c|c|c|}
\hline \multirow[t]{2}{*}{ Artículo } & \multirow[t]{2}{*}{ Comparador } & \multicolumn{4}{|c|}{ Medidas estadísticas } & \multicolumn{2}{|c|}{$\begin{array}{l}\text { Eventos del total } \\
(\%)\end{array}$} \\
\hline & & HR & \multicolumn{2}{|c|}{ IC $95 \%$} & p para & NACO & Warfarina \\
\hline \multirow{2}{*}{$\begin{array}{l}\text { Halperin et } \\
\text { al., } 2014 \\
{[13]}\end{array}$} & $\begin{array}{l}\text { Rivaroxabán en } \\
\text { edad } \geq 75\end{array}$ & 0,80 & 0,50 & 1,28 & 0,2654 & 0,66 & 0,83 \\
\hline & $\begin{array}{l}\text { Rivaroxabán en } \\
\text { edad }<75\end{array}$ & 0,54 & 0,33 & 0,89 & 0,2654 & 0,37 & 0,68 \\
\hline \multirow{3}{*}{$\begin{array}{l}\text { Halvorsen } \\
\text { et al., } 2014 \\
\text { [14] }\end{array}$} & $\begin{array}{l}\text { Apixabán en edad }< \\
65\end{array}$ & 0,87 & 0,43 & 1,74 & 0,20 & 0,31 & 0,35 \\
\hline & $\begin{array}{l}\text { Apixabán en edad } \\
65<75\end{array}$ & 0,35 & 0,20 & 0,60 & 0,20 & 0,28 & 0,81 \\
\hline & $\begin{array}{l}\text { Apixabán en edad } \geq \\
75\end{array}$ & 0,34 & 0,20 & 0,57 & 0,20 & 0,43 & 1,29 \\
\hline
\end{tabular}

NACO:nuevos anticoagulantes orales.

\section{Accidente cerebrovascular hemorrágico}

Se encontró un estudio que evaluó el riesgo de ACVH en el adulto mayor, el de Halperin et al. [12], que no evidenció diferencia en el riesgo de presentar este evento con el uso de warfarina vs. rivaroxabán en mayores de 75 años de edad (HR: 0,70; IC 95 \%: 0,39-1,25; *p: 0,37). *p para interacción. Estos datos se encuentran en la tabla 9.

Tabla 9. Accidente cerebrovascular hemorrágico en el adulto mayor

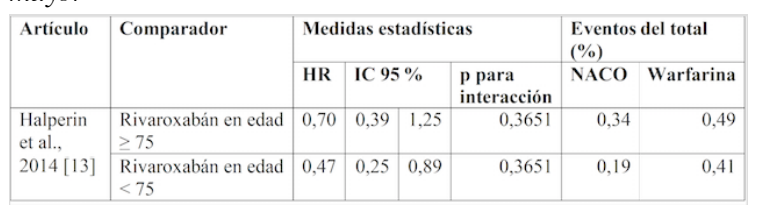

NACO:nuevos anticoagulantes orales.

Subgrupos poblacionales: antecedente de falla renal

\section{Hemorragia intracraneana}

Se encontró un estudio que evaluó este desenlace, el de Hijazi et al. [14], que reportó menor riesgo de hemorragia intracraneana con el uso de dabigatrán de $110 \mathrm{mg}$, al compararlo con warfarina, con tasa de filtración glomerular 
entre 50 y $80 \mathrm{ml} / \mathrm{min}$ (Cockroft-Gault HR: 0,29; IC $95 \%$ : 0,16-0,52; *p: 0,40. CKD-EPI HR: 0,30; IC $95 \%$ : 0,18-0,50; *p: 0,27), dabigatrán de 110 mg con una tasa de filtración glomerular menor a $50 \mathrm{ml} / \mathrm{min}$ (Cockroft-Gault HR: 0,40; IC 95 \%: 0,20-0,80; *p: 0,40. CKD-EPI HR: 0,45; IC $95 \%$ : 0,19-1,04; *p: 0,27), dabigatrán de 150 $\mathrm{mg}$ con tasa de filtración glomerular entre 50 y $80 \mathrm{ml} / \mathrm{min}$ (Cockroft-Gault HR: 0,45; IC 95 $\%: 0,27-0,74 ;{ }^{*} \mathrm{p}: 0,69$. CKD-EPI HR: 0,43; IC $95 \%: 0,27-0,68 ; *$ p: 0,96), dabigatrán de 150 mg con tasa de filtración glomerular menor a $50 \mathrm{ml} / \mathrm{min}$ (Cockroft-Gault HR: 0,31; IC $95 \%$ : 0,14-0,66; *p: 0,69. CKD-EPI HR: 0,38; IC 95 \%: 0,16-0,92; *p: 0,96). Esta información se encuentra contenida en la tabla 10 (*p para interacción).

Tabla 10. Hemorragia intracraneana en pacientes con antecedente de falla renal

\begin{tabular}{|c|c|c|c|c|c|c|c|}
\hline \multirow{2}{*}{ Artículo } & \multirow{2}{*}{ Comparador } & \multicolumn{4}{|c|}{ Medidas estadísticas } & \multicolumn{2}{|c|}{ Eventos del total (\%) } \\
\hline & & HR & & & ara interace & NACO & Warfarina \\
\hline \multirow{14}{*}{ Hijaziet al., } & \multicolumn{7}{|c|}{ Dabigatrán de $110 \mathrm{mg}$} \\
\hline & $\geq 80 \mathrm{ml} / \mathrm{min}^{*}$ & 0,13 & 0,03 & 0,57 & 0,4022 & 0,05 & 0,38 \\
\hline & $50<80 \mathrm{ml}^{2} \mathrm{~min}^{*}$ & 0,29 & 0,16 & 0,52 & 0,4022 & 0,25 & 0,87 \\
\hline & $<50 \mathrm{ml} / \mathrm{min}^{*}$ & 0,4 & 0,2 & 0,8 & 0,4022 & 0,49 & 1,23 \\
\hline & $\geq 80 \mathrm{~m} / \mathrm{min}^{* *}$ & 0,08 & 0,01 & 0,58 & 0,2745 & 0,04 & 0,51 \\
\hline & $50<<80 \mathrm{ml} / \mathrm{min}^{* * *}$ & 0,3 & 0,18 & 0,5 & 0,2745 & 0,26 & 0,85 \\
\hline & $<50 \mathrm{~m} / \mathrm{min}^{* *}$ & 0,45 & 0,19 & 1,04 & 0,2745 & 0,37 & 0,82 \\
\hline & \multicolumn{7}{|c|}{ Dabigatrán de $150 \mathrm{mg}$} \\
\hline & $\geq 80 \mathrm{~m} / \mathrm{min}{ }^{*}$ & 0,46 & 0,19 & 1,13 & 0,693 & 0,18 & 0,38 \\
\hline & $50 .<80 \mathrm{ml} / \mathrm{min}^{*}$ & 0,45 & 0,27 & 0,74 & 0,693 & 0,39 & 0,87 \\
\hline & $<50 \mathrm{ml} / \mathrm{min}^{*}$ & 0,31 & 0,14 & 0,66 & 0,693 & 0,38 & 1,23 \\
\hline & $\geq 80 \mathrm{~m} / \mathrm{min}^{* *}$ & 0,38 & 0,14 & 1,07 & 0,9635 & 0,19 & 0,51 \\
\hline & $50,<80 \mathrm{ml} / \mathrm{min}^{* * 8}$ & 0,43 & 0,27 & 0,68 & 0,9635 & 0,37 & 0,85 \\
\hline & $<50 \mathrm{ml} / \min ^{* *}$ & 0,38 & 0,16 & 0,92 & 0,9635 & $0,31 \%$ & 0,82 \\
\hline
\end{tabular}

NACO:nuevos anticoagulantes orales.

*Función renal de acuerdo con

la fórmula de Cockroft-Gault.

**Función renal de acuerdo

con la fórmula de CKD-EPI.

Subgrupos poblacionales: antecedente de patología cardiaca

\section{Hemorragia intracraneana}

Se encontraron tres artículos que evaluaron hemorragia intracraneana en pacientes con antecedente de patología cardiaca. Ferreira et al. [15] hallaron un menor riesgo con el uso de dabigatrán de $110 \mathrm{mg}$ (HR: 0,34; IC 95 \%: 0,14-0,80; *p: 0,72) y dabigatrán de 150 mg (HR: 0,39; IC $95 \%$ : 0,17-0,89; *p: 0,89) en pacientes con falla cardiaca. Nagarakanti et al. [16] mostraron menor riesgo hemorrágico con dabigatrán de $110 \mathrm{mg}$ (HR: 0,19; IC 95 \%: 0,11-0,35; *p: 0,11) y dabigatrán de 150 mg (HR: 0,36; IC $95 \%$ : 0,23-0,57; *p: 0,15) en pacientes con hipertensión arterial. Bahit et al. [17] evidenciaron menor riesgo de hemorragia intracraneana con apixabán (HR: 0,36; IC $95 \%$ : 0,20-0,66; *p: 0,59) en pacientes con enfermedad coronaria (*p para interacción). Los datos descritos se encuentran en la tabla 11.

Tabla 11. Hemorragia intracraneana en pacientes con antecedente de patología cardiaca

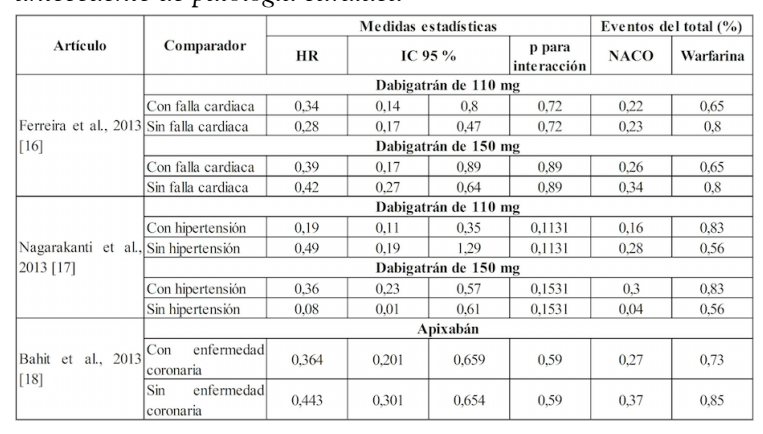

NACO:nuevos anticoagulantes orales.

\section{Conclusiones}

La importancia de la prevención de eventos hemorrágicos en la población con fibrilación auricular que requiere manejo anticoagulante ha sido descrita extensamente [18]. A partir de esta revisión es posible concluir que existe una menor prevalencia de hemorragia intracraneana y de sus diferentes subtipos en pacientes en manejo con nuevos anticoagulantes orales al compararlos con warfarina. Este estudio tiene algunas limitaciones: se debe anotar que la búsqueda, al ser llevada a cabo únicamente en dos bases de datos, podría estar excluyendo artículos de interés para el análisis. Así mismo, se debe tomar en cuenta que los criterios a través los cuales se midió la presencia de hemorragia intracraneana y sus subtipos en los distintos estudios evaluados genera dificultad en la comparación de datos entre artículos. Sin embargo, se debe resaltar que la presente revisión incluye el estudio comparativo de cuatro moléculas enmarcadas en el grupo de los nuevos anticoagulantes orales y adicionalmente evalúa desenlaces en población general y en distintos subgrupos 
poblacionales. Los resultados obtenidos son consistentes en todas las poblaciones de pacientes evaluados en los estudios. Sin embargo, es necesario conocer que, para el caso de subpoblaciones, los resultados parecen tener menor impacto estadístico al compararlos con aquellos encontrados en población general, por las diferencias entre las moléculas evaluadas, información que podría apoyar al clínico en la toma de decisiones acerca de la elección del tipo de anticoagulante en escenarios clínicos específicos. Futuros estudios sobre el tema son necesarios con el fin de evaluar la aplicabilidad de los resultados obtenidos en nuestro estudio.

\section{Referencias}

1. Castaño-Guerra $\mathrm{R}$ de J, Franco-Vergara BC, Baca-López FM, Avilés-Valverde J, González-Aceves EN, González-Hermosillo JA, et al. Guía de práctica clínica: diagnóstico y tratamiento de la fibrilación auricular. Rev Med Inst Mex Seguro Soc. 2012;50(2):213-31.

2. Roel V, Moukarzel JA, Zaidel E, Galli M, Rosa W da, Leiva R, et al. Estrategias antitrombóticas en fibrilación auricular. Registro CONAREC XIX. Rev Argent Cardiol. 2015;83(3):208-14.

3. Hart RG, Boop BS, Anderson DC. Oral anticoagulants and intracranial hemorrhage facts and hypotheses. Stroke. 1995 Aug 1;26(8):1471-7.

4. Granger CB, Alexander JH, McMurray JJV, Lopes RD, Hylek EM, Hanna M, et al. Apixaban versus warfarin in patients with atrial fibrillation. N Engl J Med. 2011 Sep 15;365(11):981-92.

5. Connolly SJ, Ezekowitz MD, Yusuf S, Eikelboom J, Oldgren J, Parekh A, et al. Dabigatran versus warfarin in patients with atrial fibrillation. N Engl J Med. 2009 Sep 17;361(12):1139-51.

6. Patel MR, Mahaffey KW, Garg J, Pan G, Singer DE, Hacke W, et al. Rivaroxaban versus Warfarin in Nonvalvular Atrial Fibrillation. N Engl J Med. 2011 Sep 8;365(10):883-91.
7. Giugliano RP, Ruff CT, Braunwald E, Murphy SA, Wiviott SD, Halperin JL, et al. Edoxaban versus warfarin in patients with atrial fibrillation. N Engl J Med. 2013 Nov 28;369(22):2093-104.

8. Capodanno D, Capranzano P, Giacchi G, Calvi V, Tamburino C. Novel oral anticoagulants versus warfarin in nonvalvular atrial fibrillation: a meta-analysis of 50,578 patients. Int J Cardiol. 2013 Aug 20;167(4):1237-41.

9. Mitchell SA, Simon TA, Raza S, Jakouloff $\mathrm{D}$, Orme ME, Lockhart I, et al. The efficacy and safety of oral anticoagulants in warfarin-suitable patients with nonvalvular atrial fibrillation: systematic review and meta-analysis. Clin Appl Thromb Off J Int Acad Clin Appl Thromb. 2013 Dec;19(6):619-31.

10. Hori M, Connolly SJ, Zhu J, Liu LS, Lau C$\mathrm{P}$, Pais $\mathrm{P}$, et al. Dabigatran versus warfarin: effects on ischemic and hemorrhagic strokes and bleeding in Asians and nonAsians with atrial fibrillation. Stroke J Cereb Circ. 2013 Jul;44(7):1891-6.

11. Goto S, Zhu J, Liu L, Oh B-H, Wojdyla DM, Aylward P, et al. Efficacy and safety of apixaban compared with warfarin for stroke prevention in patients with atrial fibrillation from East Asia: a subanalysis of the Apixaban for Reduction in Stroke and Other Thromboembolic Events in Atrial Fibrillation (ARISTOTLE) Trial. Am Heart J. 2014 Sep;168(3):303-9.

12. Halperin JL, Hankey GJ, Wojdyla DM, Piccini JP, Lokhnygina Y, Patel MR, et al. Efficacy and safety of rivaroxaban compared with warfarin among elderly patients with nonvalvular atrial fibrillation in the Rivaroxaban Once Daily, Oral, Direct Factor Xa Inhibition Compared With Vitamin K Antagonism for Prevention of Stroke and Embolism Trial in Atrial Fibrillation (ROCKET AF). Circulation. 2014 Jul 8;130(2):138-46.

13. Halvorsen S, Atar D, Yang $H$, De Caterina R, Erol C, Garcia D, et al. Efficacy and safety of apixaban compared with warfarin 
according to age for stroke prevention in atrial fibrillation: observations from the ARISTOTLE trial. Eur Heart J. 2014 Jul 21;35(28):1864-72.

14. Hijazi Z, Hohnloser SH, Oldgren J, Andersson U, Connolly SJ, Eikelboom JW, et al. Efficacy and safety of dabigatran compared with warfarin in relation to baseline renal function in patients with atrial fibrillation: a RE-LY (Randomized Evaluation of Long-term Anticoagulation Therapy) trial analysis. Circulation. 2014 Mar 4;129(9):961-70.

15. Ferreira J, Ezekowitz MD, Connolly SJ, Brueckmann M, Fraessdorf M, Reilly PA, et al. Dabigatran compared with warfarin in patients with atrial fibrillation and symptomatic heart failure: a subgroup analysis of the RE-LY trial. Eur J Heart Fail. 2013 Sep;15(9):1053-61.

16. Nagarakanti R, Wallentin L, Noack $H$, Brueckmann M, Reilly P, Clemens A, et al. Comparison of Characteristics and Outcomes of Dabigatran Versus Warfarin in Hypertensive Patients with Atrial Fibrillation (from the RE-LY Trial). Am J Cardiol. 2015 Oct 15;116(8):1204-9.

17. Bahit MC, Lopes RD, Wojdyla DM, Hohnloser SH, Alexander JH, Lewis BS, et al. Apixaban in patients with atrial fibrillation and prior coronary artery disease: insights from the ARISTOTLE trial. Int J Cardiol. 2013 Dec 10;170(2):215-20.

18. Wilson D, Salman RA-S, Klijn CJM, Lip GYH, Werring DJ. Intracerebral haemorrhage, atrial fibrillation, and anticoagulation. The Lancet. 2015 Oct;386(10005):1736-7. 